\title{
Size-dependent separation of polydisperse samples in a tapered microfluidic device
}

\author{
Ida Laila Ahmad ${ }^{1, *}$ and Mohd Ridzuan Ahmad ${ }^{2}$ \\ ${ }^{1}$ Department of Electronic Engineering, Faculty of Electrical and Electronic Engineering, Universiti Tun Hussein Onn Malaysia, 86400 \\ Batu Pahat, Johor, Malaysia \\ ${ }^{2}$ Department of Control and Mechatronics Engineering, Faculty of Electrical Engineering, Universiti Teknologi Malaysia, 81310 Skudai, \\ Johor, Malaysia
}

\begin{abstract}
In this paper we introduce the use of tapered microfluidic device for size-based separation of polydisperse samples using passive platform. The separation mechanism works based on hydrodynamic principle which is further amplified by sedimentation effect. The tapered structure allows for generation of gradient pressure as a result from velocity distribution. From this device, we achieved $98 \%$ purity of the samples collected from the outlets with low complexity design. Furthermore, the simulation and experimental results are in agreement whereby suggesting that different sized particles were collected at different outlets. The presented microfluidic system is useful and versatile due to its simplicity and ease of operation.
\end{abstract}

\section{Introduction}

Separation of micro-particles is important in various industries for example food processing, biological analysis, mineral synthesis and environmental [1]. For example, separation of harmful bacteria and unwanted microbes during fermentation process can eliminate the chances of spoiled processed food [2]. While fractionation of human blood to its components such as red blood cell (RBC) and platelets may help to provide early disease diagnosis related to blood especially blood hereditary problems, blood clotting, anaemia and it can also be used for circulating tumour cells (CTC) detection [3]. The advancements in microfluidic technology facilitate progress in various developments of separation devices. Microfluidic based separation device offers vast advantages for example it uses less reagents, reduced costs, operates at low power, uses smaller sample volumes, small and portable, flexible and allow for integration with other modules and with minimum operator handling [4], [5].

Passive separation techniques work using fluid manipulation or particle interaction for example field flow fractionation (FFF) [6], [7], filtration [8], hydrodynamic [9]-[11] and sedimentation [12]. It serves as the most fundamental approach and broadly investigated. Microfluidic based hydrodynamic separation manipulates fluid flow and suitable for separation based on size and density. It offers simple design and straightforward operation. In addition, the devices have been tested for separation of both nonbiological and biological particles such as microbeads, polymer, colloids, cancer cells and RBC [13], [14].
Although the techniques have been tested and proven to be efficient, it still suffers from being slow, need for sheath fluid and need for longer channel dimensions [7], [15], [16]. In this study, we describe a simple passive separation device for separation of polydisperse samples based on size. The device performs the separation by utilizing hydrodynamic properties of fluid and improves the samples purity through sedimentation effect resultant from the tapered microfluidic design.

This paper is organized as follows: Section 2 describes the methodology used which includes device design, working principles, finite element simulation, device fabrication and experimental procedures. In Section 3, the results obtained from both simulation and experiments were discussed thoroughly. A fair comparisons have been carried out and the device performance have been evaluated.

\section{Material and method}

\subsection{Device design}

The use of tapered design microfluidic were widely adopted by other researchers for various purpose; stiffness measurements, deformability detection and retention time [17]-[19]. However, the applicability of the design for separation applications has yet to be reported. The design of tapered microfluidic device consists of a single inlet without any sheath inlet and three separation outlets as shown in Figure 1. Length of tapered area is $4 \mathrm{~mm}$, diameter of inlet and outlets are 2 $\mathrm{mm}$ and the height is $10 \mu \mathrm{m}$. Four devices with different taper angles were designed with $6^{\circ}, 12^{\circ}, 20^{\circ}$ and $25^{\circ}$.

\footnotetext{
* Corresponding author: ida@uthm.edu.my
} 


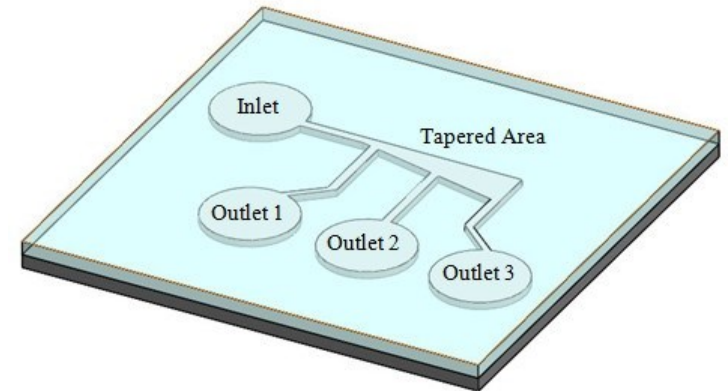

Fig. 1. Tapered microfluidic device design

The design was implemented by using an equivalent resistive circuit model to represent the resultant circuit during steady state operation as given by Figure 2 .

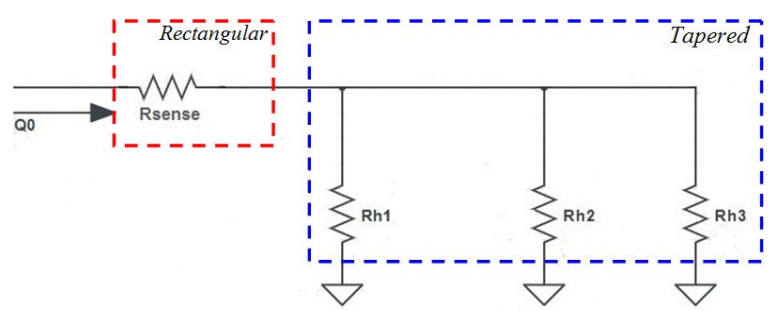

Fig. 2. Equivalent resistive circuit model

\subsection{Working principle}

Figure 3 illustrates the working principle of the proposed device.

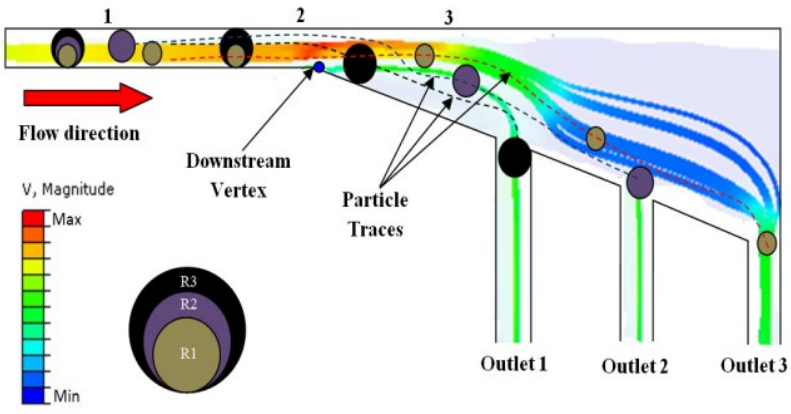

Fig. 3. Working principle of tapered microfluidic device

Considering samples of different sizes were flown into the inlet, these multi-particles will be aligned along the narrow focussing length towards the tapered area. This eliminates the need for sheath flow during particle focussing. Upon reaching at the downstream vertex, each particle starts to travel using its own trajectory which is dictated by the particle radius. The generated flow profile inside tapered microfluidic has a lower mean velocity at the central while higher velocity at the inlet and outlets created a gradient streamlines [12]. At point 3 where mean velocity is very small, gravity takes effect and sedimentation on larger particle is more significant. Larger particle tend to deviate from the original fluid path and try to escape using the nearest outlet (Outlet 1). On the contrary, smaller particle will follow the fluid path and will travel towards the lowest hydrodynamic resistance outlet (Outlet 3) [15].
Therefore, successful separation can be observed with largest particles collected at Outlet 1 and smallest particles collected at Outlet 2. Hypothetically, the intermediate sized particles will be collected at the Outlet 2. To understand this phenomena better, it is basically controlled by Reynolds number and Stokes number. These dimensionless parameters determine the laminarity profile of the fluid flow inside tapered microfluidic and the behaviour of particles moving inside the fluidic environment [20]. Larger particles will have higher Stoke number causing them to sediment faster as compared to its smaller counterparts. Stokes number can be described as the following equation:

$$
S t=\frac{\tau_{r}}{\tau_{f}}=\frac{\rho_{p} a^{2} / 18 \eta}{D_{h} / V}
$$

Where $\rho_{p}$ is the particle density, $a$ is the diameter, $\eta$ is the kinematic viscosity of fluid, $V$ is velocity and $D_{h}$ is the hydraulic diameter [21].

\subsection{Finite element simulation}

In this research, Abaqus 6.12 developed by Dassaults Systemes Simulia Corp, Providence, RI, USA was used. For simulation purpose, a tapered microchannel was designed using Eulerian material and was assigned with water properties. Hexahedral meshing was used and different boundary conditions were assigned at the respected inlet (inlet velocity) and outlets (free flow) as shown in Figure 4. Finite element models were developed for both polydisperse samples containing polystyrene microbeads and human cervical epithelial carcinoma (HeLa) cells. Deformable parts were meshed with tetrahedral elements and materials assignment were defined according to polystyrene microbeads and HeLa properties comes from previous literature [22]. Figure 5 shows the developed model. Finally, Figure 6 shows the assembly setting for simulation condition.

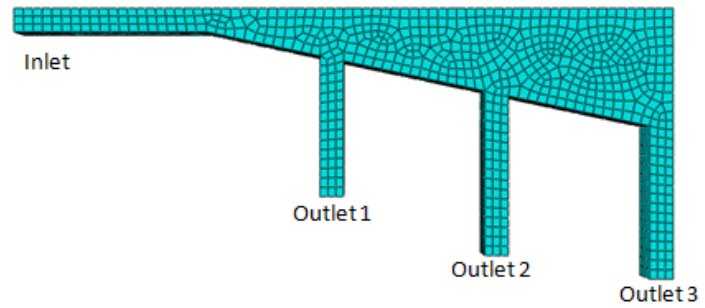

Fig. 4. Hexahedral meshing for tapered microfluidic device

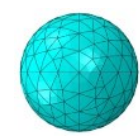

Fig. 5. Tetrahedral meshing for polystyrene microbeads and HeLa cell 


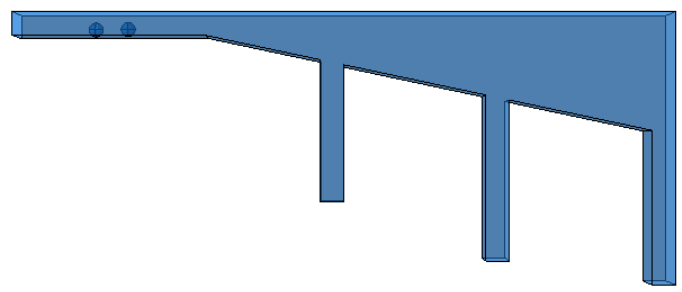

Fig. 6. Assembly setting for finite element simulation consists of two particles representing polydisperse samples

\subsection{Device fabrication}

Tapered design for the microchannel was drawn using LayoutEditor software. A 2.5 inch glass chrome mask was used to transfer the design by using a direct laser writing technique on $\mu$ PG machine (Heidelberg Instruments, Germany). Master mould was prepared using a silicone wafer as the substrate. Negative photoresist SU8-3005 (Kayaku Microchem, Japan) was poured onto the substrate and spun using a spin coater for even coating. The spinning speed were programmed at $500 \mathrm{rpm}$ for 10 seconds and followed with $4000 \mathrm{rpm}$ for 30 seconds.

Then, the silicone substrate was put onto a hot plate for soft baked at $95^{\circ} \mathrm{C}$ for 3 minutes. Following this, a mask aligner was used to align the chrome mask and the substrate for design pattern transfer via photolithography technique whereby ultraviolet (UV) exposure was set to intensity level of $200 \mathrm{~mJ} / \mathrm{cm}$ for 11.8 seconds. Two stages post-baked procedures were conducted by setting the temperature at $65^{\circ} \mathrm{C}$ for 1 minute and $95^{\circ} \mathrm{C}$ for 3 minutes to harden the substrate. Etchant was used to etch away the unexposed SU-8 layer and further developed using SU-8 developer (Microchem, Newton, MA) leaving a positive relief containing microchannel pattern. Isopropyl alcohol (IPA) was used to further clean the master mould.

The usage of liquid polydimethylsiloxane (PDMS) is widely adopted for various microfluidic applications. PDMS generally is inexpensive, flexible and optically transparent [23]. It is also biocompatible and highly permeable to carbon dioxide $\left(\mathrm{CO}_{2}\right)$ and oxygen $\left(\mathrm{O}_{2}\right)$ allowing for biological assays and cell culturing experiments. Oxidation of PDMS channels can create high surface energy allowing polar liquids like water to easily fill the channel. In order to fabricate the PDMS device, the liquid PDMS pre-polymer and curing agent (SILPOT 184, SILPOT CAT Dow Corning, USA) were mixed in the ratio of 9:1. After that, air bubble removal and degassed procedures were carried out and the mixture was poured over the master mould. For a good cross-linking effect the mixture was cured in a convection oven at $65^{\circ} \mathrm{C}$ for 2 hours. The cured PDMS was peeled from the master leaving the negative cast of the microchannel pattern. Inlets and outlets were punched manually using $2 \mathrm{~mm}$ puncher (Harris, Redding, CA). Plasma oxidation treatment was done at $20 \mathrm{~mA}$ for 3 minutes to seal the PDMS with glass slide. Tygon tubings were connected to the outlets and glued to plastic vials secured at adjustable $\mathrm{X}-\mathrm{Y}$ manipulator for output collection. Figure 7 shows the completed PDMS chip.

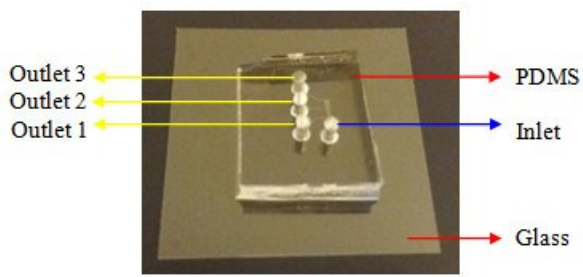

Fig. 7. Completed PDMS chip for tapered microfluidic device

\subsection{Experimental setup}

To demonstrate the operation of fabricated device non fluorescent polystyrene (PS) microbeads with diameter of $3 \mu \mathrm{m}$ (Polysciences. Inc.) and HeLa cells were used as samples. Next, HeLa cells were prepared following cell harvesting procedures. The cell tube contained $5 \times 10^{5}$ of cells $/ \mathrm{mL}$ was thawed using water bath and cell culturing was prepared inside clean bench. After culturing process was done, the remaining cell was kept in an incubator at $37^{\circ} \mathrm{C}$ with $\mathrm{CO}_{2}$ level 5 . Cell viability was checked under confocal microscope before usage. A mixture containing PS microbeads and HeLa cells were prepared and diluted further then centrifuged before re-suspended in culture medium.

During experiment, KDS Scientific syringe pump was connected to the inlet and was given a constant flow rate throughout the experiment. Phosphate Buffer Saline (PBS) or culture medium solutions were introduced into the microchannel first to eliminate unwanted bubbles. Then, the samples were introduced to the inlet. The separation behaviour was recorded using high speed charge-coupled-device (CCD) camera (Hamamatsu ORCA ER) and inverted microscope (IX-71 Olympus Corp., Japan).

\section{Results and discussions}

\subsection{Finite element simulation results}

Polydisperse separation simulation results are shown on Table 1 below.

Table 1. Separation simulation results

\begin{tabular}{|c|l|l|}
\hline $\begin{array}{c}\text { Device } \\
\text { angle }\left(^{\circ}\right)\end{array}$ & $\begin{array}{c}\text { Monodisperse } \\
\text { samples }\end{array}$ & Polydisperse samples \\
\hline 6 & No separation & No separation \\
\hline 12 & No separation & No separation \\
\hline 20 & No separation & $\begin{array}{l}\text { Outlet 1: HeLa cells } \\
\text { Outlet 2: None } \\
\text { Outlet 3: PS Microbeads }\end{array}$ \\
\hline 25 & No separation & $\begin{array}{l}\text { Outlet 1: HeLa cells } \\
\text { Outlet 2: None } \\
\text { Outlet 3: PS Microbeads }\end{array}$ \\
\hline
\end{tabular}


Based on the simulation results, successful separation can be seen with the use of larger taper angles devices $\left(20^{\circ}\right.$ and $\left.25^{\circ}\right)$. Larger angles provide more widening and significantly enhanced the sedimentation effect experienced by larger particles [12]. As a result, the larger particle (HeLa) will be collected at the Outlet 1. This phenomena also is supported by the Stoke number theory. From the calculation, St for HeLa cell is higher than the microbeads, indicating that it has the preference to travel on its own path. In comparison, hydrodynamic resistance values are the highest at the furthest outlet (Outlet 3). Smaller particle which have smaller St number will flow according to fluid streamlines moving towards Outlet 3 [15].

\subsection{Experimental results}

Prior to the separation experiment, the tapered microfluidic was tested for leaking. It was observed that any flow rate higher than $3.0 \mu \mathrm{l} / \mathrm{min}$ will cause device leakage. Therefore flow rate was set to be in between 0.5 $-3.0 \mu \mathrm{l} / \mathrm{min}$ was used during the experiment. Table 2 shows the separation results. It can be seen that the simulation results are in agreement with the experimentation results although different flow rates were used.

Table 2. Separation experiment results

\begin{tabular}{|c|c|c|}
\hline $\begin{array}{l}\text { Device } \\
\text { angle }\left({ }^{\circ}\right)\end{array}$ & $\begin{array}{c}\text { Flow rate } \\
(\mu \mathrm{l} / \mathrm{min})\end{array}$ & Polydisperse sample \\
\hline 6 & \multirow{4}{*}{$\begin{array}{l}0.5 \\
1.0 \\
1.5 \\
2.0 \\
2.5 \\
3.0\end{array}$} & No separation \\
\hline 12 & & No separation \\
\hline 20 & & $\begin{array}{l}\text { Outlet } 1: \text { HeLa cells } \\
\text { Outlet 2: None } \\
\text { Outlet 3: PS Microbeads }\end{array}$ \\
\hline 25 & & $\begin{array}{l}\text { Outlet } 1: \text { HeLa cells } \\
\text { Outlet 2: None } \\
\text { Outlet 3:PS Microbeads }\end{array}$ \\
\hline
\end{tabular}

The following Figure 8(a)-(c) shows the collected samples from the three outlets. It can be seen that, HeLa cells were collected from Outlet 1, microbeads were collected from Outlet 3 and Outlet 2 was empty.

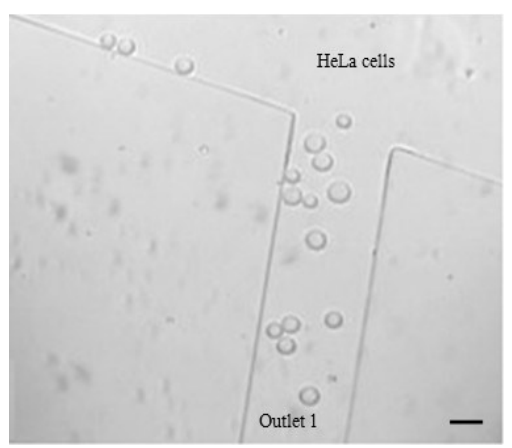

(a)

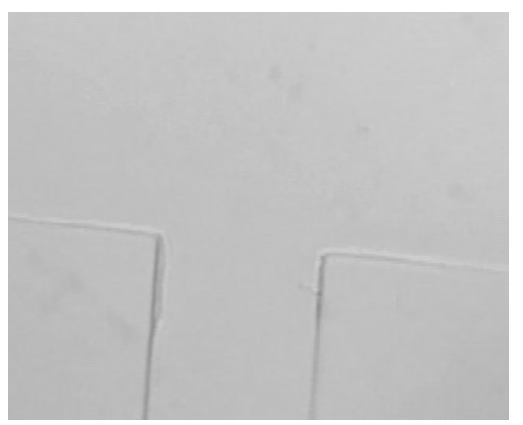

(b)

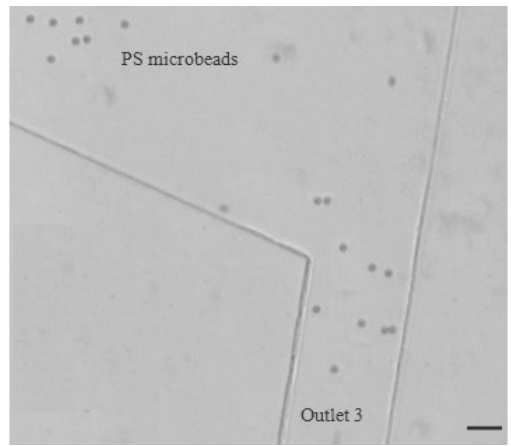

(c)

Fig. 8. Separation experiment results. (a) HeLa cells collected from Outlet 1 (b) Nothing is collected from Outlet 2 (c) Microbeads collected from Outlet 3. Scale used is $10 \mu \mathrm{m}$

Overall, the performance of the proposed microfluidic separator device is in agreement with the simulations. It further proves the co-existence between the hydrodynamic effect and sedimentation due to uniquely designed tapered microchannel. The separation behavior can be understood better by comparing how rigid (PS microbeads) and deformable particle (HeLa cells) travel inside a microfluidic channel. The comparison is listed by Table 3 below.

Table 3. Traveling behavior inside microchannel

\begin{tabular}{|c|c|c|c|}
\hline Parameter & Rigid & Deformable & Ref. \\
\hline Speed & High & Slow & {$[24]$} \\
\hline Deformation & Low & High & {$[25]$} \\
\hline Density & Light & Dense & {$[26]$} \\
\hline Nature & Hard & Soft (Fluidic) & {$[27]$} \\
\hline $\begin{array}{c}\text { Travel } \\
\text { pattern }\end{array}$ & $\begin{array}{c}\text { Travel along } \\
\text { centerline }\end{array}$ & $\begin{array}{c}\text { Travel near } \\
\text { channel wall }\end{array}$ & {$[28]$} \\
\hline
\end{tabular}

These behaviors imply that, both rigid and deformable particle possess different characteristics during traveling inside fluidic condition. Therefore, good device design and parameter tuning can be manipulated 
to achieve successful separation. After experiment, samples collections from the outlets were evaluated to test the device performance. A good microfluidic separator should meet with these three basic requirements; high purity samples, acceptable flow rate (speed) and low complexity design. Purity of samples can be translated into the following formula:

$$
\text { Purity }=\frac{\left(\mathrm{N}_{\text {Target }}\right)_{\text {Outlets }}}{\left(\mathrm{N}_{\text {Target }} \text { Waste }\right)_{\text {Outlets }}} \times 100 \%
$$

Where $\mathrm{N}_{\text {Target }}$ was the number of target particles and $\mathrm{N}_{\text {Waste }}$ was the number of waste particles [14]. The percentage of purity was calculated for all flow rates tested and the results can be seen from Figure 9 below.

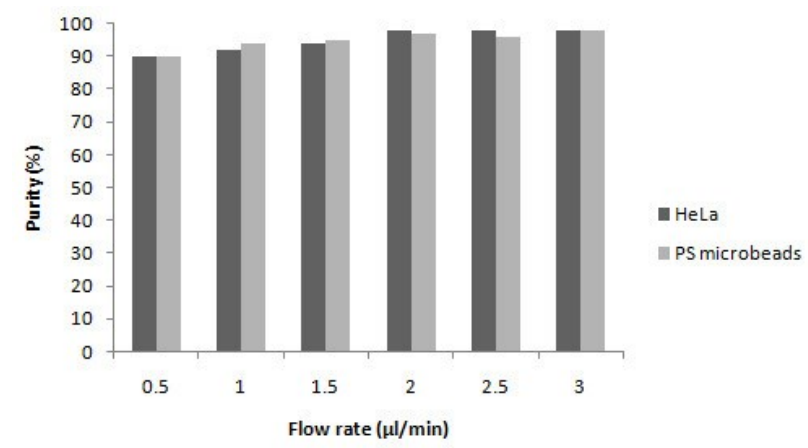

Fig. 9. Purity of samples collection for all tested flow rates

Highest purity of $98 \%$ can be achieved from the proposed device as illustrated by Figure 9. Therefore, the use of tapered microfluidic was proven to be effective in improving samples purity.

\section{Conclusion}

We have demonstrated a novel, passive tapered microfluidic device for separation of polydisperse sample containing PS microbeads and HeLa cells. The present study demonstrated high purity of samples collection at $98 \%$ for most of flow rates tested. The proposed device mechanism works by using coupling between hydrodynamic principle and sedimentation effect. Even though both techniques are well established, but the capability for polydisperse separation is demonstrated by this study. Since the proposed device dimensions are easily tunable and the operation is straight-forward, specific application development is possible. Above all, the capability to realize a label free sorting with high purity can be useful for various applications such as samples preparation and medical diagnostic

The authors wish to extend their gratitude to the financial aid sponsored by Ministry of Higher Education of Malaysia (Grant Nos. 4L640), and Universiti Teknologi Malaysia (Grant Nos. 03E11, 03G47, 4J148, 02G46 and 03H80). Special thanks for research colleagues at Micro-Nano System Engineering
Department, Nagoya University, Nagoya, Aichi, Japan (Dr. Masaru Takeuchi and AP. Dr. Masahiro Nakajima) for beneficial discussions, guidance and laboratory facilities.

\section{References}

1. P. Sajeesh and A. K. Sen, "Particle separation and sorting in microfluidic devices: A review," Microfluid. Nanofluidics, vol. 17, no. 1, pp. 1-52, 2014.

2. D. R. Gossett, W. M. Weaver, A. J. Mach, S. C. Hur, H. T. K. Tse, W. Lee, H. Amini, and D. Di Carlo, "Label-free cell separation and sorting in microfluidic systems," Anal. Bioanal. Chem., vol. 397, no. 8, pp. 3249-3267, 2010.

3. H. W. Hou, L. Wu, D. Amador-Munoz, M. P. Vera, B. D. Levy, R. M. Baron, and J. Han, "Microfluidic blood margination $(\mu \mathrm{BM})$ : A 'cell-based' blood purification platform for broad spectrum immunomodulation in murine model of sepsis," 18th Int. Conf. Miniaturized Syst. Chem. Life Sci. MicroTAS 2014, vol. 6, no. 2, pp. 333-335, 2014.

4. C. Rivet, H. Lee, A. Hirsch, S. Hamilton, and H. Lu, "Microfluidics for medical diagnostics and biosensors," Chem. Eng. Sci., vol. 66, no. 7, pp. 1490-1507, Apr. 2011.

5. A. M. Streets and Y. Huang, "Chip in a lab: Microfluidics for next generation life science research," Biomicrofluidics, vol. 7, no. 1, p. 11302 , Jan. 2013.

6. M. Seki, M. Yamada, M. Yasuda, and Y. Sai, "Continuous separation of particles using a microfluidic device equipped with flow rate control valves," J. Chromatogr. A, vol. 1127, no. 1-2, pp. 214-220, 2006.

7. J. Takagi, M. Yamada, M. Yasuda, and M. Seki, "Continuous particle separation in a microchannel having asymmetrically arranged multiple branches.," Lab Chip, vol. 5, no. 7, pp. 778-784, 2005.

8. Y. Park, C. a Best, K. Badizadegan, R. R. Dasari, M. S. Feld, T. Kuriabova, M. L. Henle, A. J. Levine, and G. Popescu, "Measurement of red blood cell mechanics during morphological changes.," Proc. Natl. Acad. Sci. U. S. A., vol. 107, no. 15, pp. 67316, Apr. 2010.

9. M. Yamada, K. Kano, Y. Tsuda, J. Kobayashi, M. Yamato, M. Seki, and T. Okano, "Microfluidic devices for size-dependent separation of liver cells," Biomed. Microdevices, vol. 9, no. 5, pp. 637-645, 2007.

10. D. W. Inglis, J. a Davis, R. H. Austin, and J. C. Sturm, "Critical particle size for fractionation by deterministic lateral displacement.," Lab Chip, vol. 6, no. 5, pp. 655-658, 2006.

11. J. P. Beech, S. H. Holm, K. Adolfsson, and J. O. Tegenfeldt, "Sorting cells by size, shape and deformability," Lab Chip, vol. 12, no. 6, p. 1048, 2012. 
12. D. Huh, J. H. Bahng, Y. Ling, H. H. Wei, O. D. Kripfgans, J. B. Fowlkes, J. B. Grotberg, and S. Takayama, "Gravity-driven microfluidic particle sorting device with hydrodynamic separation amplification," Anal. Chem., vol. 79, no. 4, pp. 1369-1376, 2007.

13. J. Alvankarian, A. Bahadorimehr, and B. Yeop Majlis, "A pillar-based microfilter for isolation of white blood cells on elastomeric substrate," Biomicrofluidics, vol. 7, no. 1, p. 014102(1)014102(16), 2013.

14. Y. Y. Chiu, C. K. Huang, and Y. W. Lu, "Enhancement of microfluidic particle separation using cross-flow filters with hydrodynamic focusing," Biomicrofluidics, vol. 10, no. 1, p. 011906(1)-011906(13), 2016.

15. Z. Wu and K. Hjort, "Microfluidic Hydrodynamic Cell Separation: A Review," Micro Nanosyst., vol. 1, no. 3, pp. 181-192, 2009.

16. D. Di Carlo, J. F. Edd, D. Irimia, R. G. Tompkins, and $\mathrm{M}$. Toner, "Equilibrium separation and filtration of particles using differential inertial focusing," Anal. Chem., vol. 80, no. 6, pp. 2204-2211, 2008.

17. C.-H. D. Tsai, M. Kaneko, S. Sakuma, and F. Arai, "Observability of cell stiffness in micro-channel method," 2013 IEEE Int. Conf. Robot. Autom., pp. 2807-2813, May 2013.

18. G. Guan, L. Wu, A. A. Bhagat, Z. Li, P. C. Y. Chen, S. Chao, C. J. Ong, and J. Han, "Spiral microchannel with rectangular and trapezoidal cross-sections for size based particle separation.," Sci. Rep., vol. 3, no. C, p. 1475, 2013.

19. Y. N. Luo, D. Y. Chen, Y. Zhao, C. Wei, X. T. Zhao, W. T. Yue, R. Long, J. B. Wang, and J. Chen, "A constriction channel based microfluidic system enabling continuous characterization of cellular instantaneous Young's modulus," Sensors Actuators B Chem., vol. 202, pp. 1183-1189, Oct. 2014.

20. J. Happel and H. Brenner, Low Reynolds number hydrodynamics: with special applications to particulate media. Springer Netherlands, 2012.
21. E. Ortega-Rivas, Unit Operations of Particulate Solids: Theory and Practice. CRC Press, 2016.

22. K. Tomankova and P. Kolar, Mechanical characterisation of HeLa cells using atomic force microscopy. 2012.

23. S. K. Sia and G. M. Whitesides, "Microfluidic devices fabricated in poly(dimethylsiloxane) for biological studies," Electrophoresis, vol. 24, no. 21. pp. 3563-3576, 2003.

24. S. Huang, H. Bow, M. Diez-silva, and J. Han, "Applying A Microfluidic 'Deformability Cytometry' To Measure Stiffness of Malaria Infected Red Blood Cells at Body and Febrile Temperatures," in 14th International Conference on Miniaturized Systems for Chemistry and Life Sciences, 2010, no. October, pp. 259-261.

25. E. M. Nascimento, N. Nogueira, T. Silva, T. Braschler, N. Demierre, P. Renaud, and A. G. Oliva, "Dielectrophoretic sorting on a microfabricated flow cytometer: Label free separation of Babesia bovis infected erythrocytes," Bioelectrochemistry, vol. 73, no. 2, pp. 123-128, 2008.

26. J. Rousselet, G. H. Markx, and R. Pethig, "Separation of erythrocytes and latex beads by dielectrophoretic levitation and hyperlayer fieldflow fractionation," in Colloids and Surfaces A: Physicochemical and Engineering Aspects, 1998, vol. 140, no. 1-3, pp. 209-216.

27. A. A. S. Bhagat, S. S. Kuntaegowdanahalli, and I. Papautsky, "Enhanced particle filtration in straight microchannels using shear-modulated inertial migration," Phys. Fluids, vol. 20, no. 10, p. 101702, 2008.

28. S. Kuntaegowdanahalli and A. Bhagat, "Inertial microfluidics for continuous particle separation in spiral microchannels," Lab Chip, vol. 9, no. 20, pp. 2973-80, 2009. 